\title{
Subchronic and Reproductive/Developmental Toxicity Studies of Tetrahydrocurcumin in Rats
}

\author{
Muhammed Majeed, Sankaran Natarajan, Anjali Pandey, Sarang Bani and Lakshmi Mundkur \\ Sami Labs Limited, Karnataka, India
}

\begin{abstract}
Tetrahydrocurcumin (THC) is a major metabolite of curcumin, which is obtained from Curcuma longa. THC has various benefits and overcomes the bioavailability issue of curcumin. To establish it as a pharmacologically active molecule, its safety profile has to be determined. Thus, the present study aimed to determine the preclinical safety profile of THC in a 90-day subchronic and reproductive/developmental toxicity study in Wistar rats. THC at oral doses of 100, 200, and $400 \mathrm{mg} / \mathrm{kg}$ was administered daily for 90 days. Rats in the recovery group were kept for 14 days after treatment termination. The animals were observed for treatment-related morbidity, mortality, and changes in clinical signs, clinical pathology, and histopathology. In the reproductive/developmental toxicity study, THC at 100,200 , and $400 \mathrm{mg} / \mathrm{kg}$ was administered orally to rats and the reproductive/developmental parameters in adult male and female rats and pups were observed. THC at up to $400 \mathrm{mg} / \mathrm{kg} / \mathrm{day}$ of did not have any significant effect on all parameters in male and female rats in both toxicity studies. Thus, $400 \mathrm{mg} / \mathrm{kg} / \mathrm{day}$ can be considered as the no-observed-adverse-effect-level of THC in rats.
\end{abstract}

Key words: Tetrahydrocurcumin, Toxicity, Subchronic, Reproductive/developmental

\section{INTRODUCTION}

Curcuma longa, (family Zingiberaceae) also known as turmeric, is a perennial herb (1). Turmeric powder prepared from its rhizomes is used as a coloring and flavoring agent. Moreover, it has been used for over 4,000 years for religious purposes in the historical Vedic religion $(2,3)$.

Turmeric contains curcumin (Fig. 1), which is biologically active and yellow (1). The quality, medicinal use, and even economic value of turmeric are determined by its curcumin content (4). Curcumin is a safe antioxidant with a wide range of therapeutic use (5). It is an established nutritional supplement with antioxidant, anticancer, antiinflammatory, and antimicrobial activities. It is also effec-

Correspondence to: Anjali Pandey, Sami Labs Limited, \#19/1, 19/ 2, I Main, II Phase, Peenya Industrial Area, Bangalore 560 058, Karnataka, India

E-mail: anjalipandey@samilabs.com

This is an Open-Access article distributed under the terms of the Creative Commons Attribution Non-Commercial License (http:// creativecommons.org/licenses/by-nc/3.0) which permits unrestricted non-commercial use, distribution, and reproduction in any medium, provided the original work is properly cited. tive for Alzheimer's disease, cystic fibrosis, rheumatoid arthritis, cataract, and stress and cholesterol management (1).

Tetrahydrocurcumin (THC) (Fig. 1) is a major metabolite of curcumin, along with hexahydrocurcumin and octahydrocurcumin (6-9). Curcumin is converted to THC in<smiles>COc1cc(/C=C/C(=O)CC(=O)/C=C/c2ccc(O)c(OC)c2)ccc1O</smiles><smiles>CCOCCOCC</smiles>

Fig. 1. Structure of curcumin and tetrahydrocurcumin. 
two steps by NADPH-dependent curcumin/dihydrocurcumin reductase enzyme, with dihydrocurcumin as the intermediate product (10).

THC, which is white, can replace synthetic antioxidants in the formulation of colorless cosmetics and food products. It exhibits superoxide-scavenging activity, and decreases the deterioration of various lipid components by inhibiting lipid oxidation (7). In addition, THC overcomes the low bioavailability of curcumin, as it was reported that in mouse plasma, THC is detected $80 \mathrm{~min}$ post administration, whereas curcumin is not (11).

Curcumin has half-lives of 186 and $813 \mathrm{~min}$, whereas those of THC were 111 and $232 \mathrm{~min}$, showing that THC is more stable than curcumin (12). THC is more stable than curcumin in $0.1 \mathrm{M}$ phosphate buffer at $\mathrm{pH} 7.2$ and $37^{\circ} \mathrm{C}$ (11). In addition, the biological effect of curcumin is influenced by the chemical stability of THC (13). THC binds phospholipase A2 more potently than curcumin because the hydrocarbon chain of THC lacks double bond at both ends of the aromatic rings (14). Moreover, the antioxidant, anti-inflammatory, antidiabetic, antihyperlipidemic, and antihypertensive effects of THC are more potent than those of curcumin. Furthermore, THC reduces the accumulation and cross-linking of collagen and prevents azoxymethane-induced colon cancer to a greater extent than curcumin (13-20). THC also suppresses lipid peroxidation in erythrocyte ghosts, radiation-induced lipid peroxidation, nitrilotriacetate-induced oxidative renal damage, and LDL oxidation more potently than curcumin (21-24).

The biological effects of THC have been reported by several studies (13-27), but its toxicity profile has not been shown. Thus, the present study was conducted to determine the preclinical safety profile of THC using a 90-day subchronic and reproductive/developmental toxicity study in Wistar rats. During efficacy study of THC in the experimental animals $40 \mathrm{mg} / \mathrm{kg}$ PO was obtained as efficacy dose, considering that $400 \mathrm{mg} / \mathrm{kg}$ was taken up for the toxicity studies.

\section{MATERIALS AND METHODS}

Chemicals. THC is a white derivative of curcumin and it contains no $\alpha, \beta$-unsaturated carbonyl moiety (12). THC was prepared in an inert solvent using palladium as a metallic catalyst. During the process, the selective double bond of curcumin was hydrogenated, thereby forming THC (14). The resulting THC was then identified using high performance liquid chromatography with UV/PDA detector at $280 \mathrm{~nm}$ and standardized to $95 \%$ for further use. THC has a chemical formula of 1,7-Bis(4-hydroxy-3methoxyphenyl)-3,5-heptanedione, molecular formula of $\mathrm{C}_{21} \mathrm{H}_{24} \mathrm{O}_{6}$, molecular weight of 372.4117 , and melting range of $97-99^{\circ} \mathrm{C}(8)$.

The three-dimensional crystal structure of THC has been studied by X-ray crystallography. The molecule is non-planar and the benzene rings positioned at the end of heptane chains are orthogonally placed, with a dihedral angle of $84.09^{\circ}$, which means that the benzene rings are perpendicular to each other. The molecular geometry and $\mathrm{H}$-atom locations reveal that a heptane-3,5-dione moiety exists in the keto-enol form, with the enolic hydroxyl Hatom equally disordered between the O-3 and O-5 dione moiety. Of the possible tautomeric forms, in the crystal phase, $\beta$-diketones prefer the cis-enol arrangement stabilized by strong intramolecular hydrogen bond. THC is off white in color, insoluble in water, and soluble in organic solvents, such as acetone, ethyl acetate, chloroform, and isopropanol (28). Carboxymethyl cellulose is used as a vehicle in both the 90-day subchronic and reproductive/ developmental toxicity studies.

Animals. Adult Wistar rats, 50 and 40 each of either sex, were used for the 90-day subchronic and reproductive/developmental toxicity studies, respectively. Females used were nonpregnant and nulliparous. The animals were housed in a standard propylene cage with stainless-steel grill with autoclaved corn cob beddings, with two rats per

Table 1. Dose grouping of the subchronic toxicity study

\begin{tabular}{|c|c|c|c|c|c|}
\hline Group no. & Treatment groups & Dose (mg/kg/day) & Concentration $(\mathrm{mg} / \mathrm{mL})$ & Sex & No. of rats \\
\hline \multirow[t]{2}{*}{ G1 } & Control & 0 & NA & $M$ & 10 \\
\hline & & & & $\mathrm{F}$ & 10 \\
\hline \multirow[t]{2}{*}{ G2 } & Low dose & 100 & 10 & M & 10 \\
\hline & & & & $\mathrm{F}$ & 10 \\
\hline \multirow[t]{2}{*}{ G3 } & Mid dose & 200 & 20 & M & 10 \\
\hline & & & & $\mathrm{F}$ & 10 \\
\hline \multirow[t]{2}{*}{ G4 } & High dose & 400 & 40 & M & 10 \\
\hline & & & & $\mathrm{F}$ & 10 \\
\hline \multirow[t]{2}{*}{ G1R* } & Control & 0 & NA & M & 5 \\
\hline & & & & $\mathrm{F}$ & 5 \\
\hline \multirow[t]{2}{*}{ G4R* } & High dose & 400 & 40 & M & 5 \\
\hline & & & & $\mathrm{F}$ & 5 \\
\hline
\end{tabular}

M-male, F-female, NA-not available, *Recovery group. 
sex per cage. During the acclimatization, experimental, and recovery periods, the housing room was air-conditioned with adequate fresh air supply (10-12 air changes per hour) and was maintained at a temperature of 20.2$24.9^{\circ} \mathrm{C}$ and relative humidity of $51-59 \%$ under a $12-\mathrm{hr}$ light and dark cycle. Pelleted animal feed (M/s Rayans Biotechnologies Pvt. Ltd., Hyderabad, India) and purified water were provided ad libitum.

Ethics. The Institutional Animal Ethics Committee (IAEC) (registration no. VIP/IAEC/06/2015) had approved the protocols and animals used in the protocols of both the subchronic and reproductive/developmental studies. The study was conducted in a CPSEA-approved laboratory (registration no. 1683/RO/RcBiBt/13/CPCSEA) in compliance with the CPSEA and OECD 408 and 421 guidelines for testing of chemicals.

Ninety-day subchronic toxicity study. After a minimum 6 days of acclimatization, ten rats of each sex per group were assigned to the main study groups, and five rats of each sex were assigned to the recovery group. Rats in the control (G1)/control recovery (G1R), low-dose (G2), mid-dose (G3), and high-dose (G4)/high-dose recovery (G4R) groups were administered THC at $0,100,200$, and
Table 2. Dose grouping of the reproductive/developmental study

\begin{tabular}{ccclc}
\hline \hline $\begin{array}{c}\text { Group } \\
\text { no. }\end{array}$ & $\begin{array}{c}\text { Treatment } \\
\text { groups }\end{array}$ & $\begin{array}{c}\text { Dose } \\
(\mathrm{mg} / \mathrm{kg} / \mathrm{day})\end{array}$ & Sex & $\begin{array}{c}\text { No. of } \\
\text { rats }\end{array}$ \\
\hline G1 & Control & 0 & $\mathrm{M}$ & 10 \\
& & & $\mathrm{~F}$ & 10 \\
G2 & Low dose & 100 & $\mathrm{M}$ & 10 \\
& & & $\mathrm{~F}$ & 10 \\
G3 & Mid dose & 200 & $\mathrm{M}$ & 10 \\
& & & $\mathrm{~F}$ & 10 \\
G4 & High dose & 400 & $\mathrm{M}$ & 10 \\
& & & $\mathrm{~F}$ & 10 \\
\hline
\end{tabular}

M-male, F-female.

$400 \mathrm{mg} / \mathrm{kg} / \mathrm{day}$, respectively, as depicted in Table 1 .

During the study period, all animals were checked twice daily for any abnormality, morbidity, and mortality. Clinical signs were observed twice daily for the first three days of treatment and once daily thereafter throughout the experimental and recovery periods. Body weight was measured once before randomization and once weekly during the acclimatization, experimental, and recovery periods. Food consumption per cage was calculated once weekly by the weight of food left and food fed.

Blood samples were collected from the main and recov-
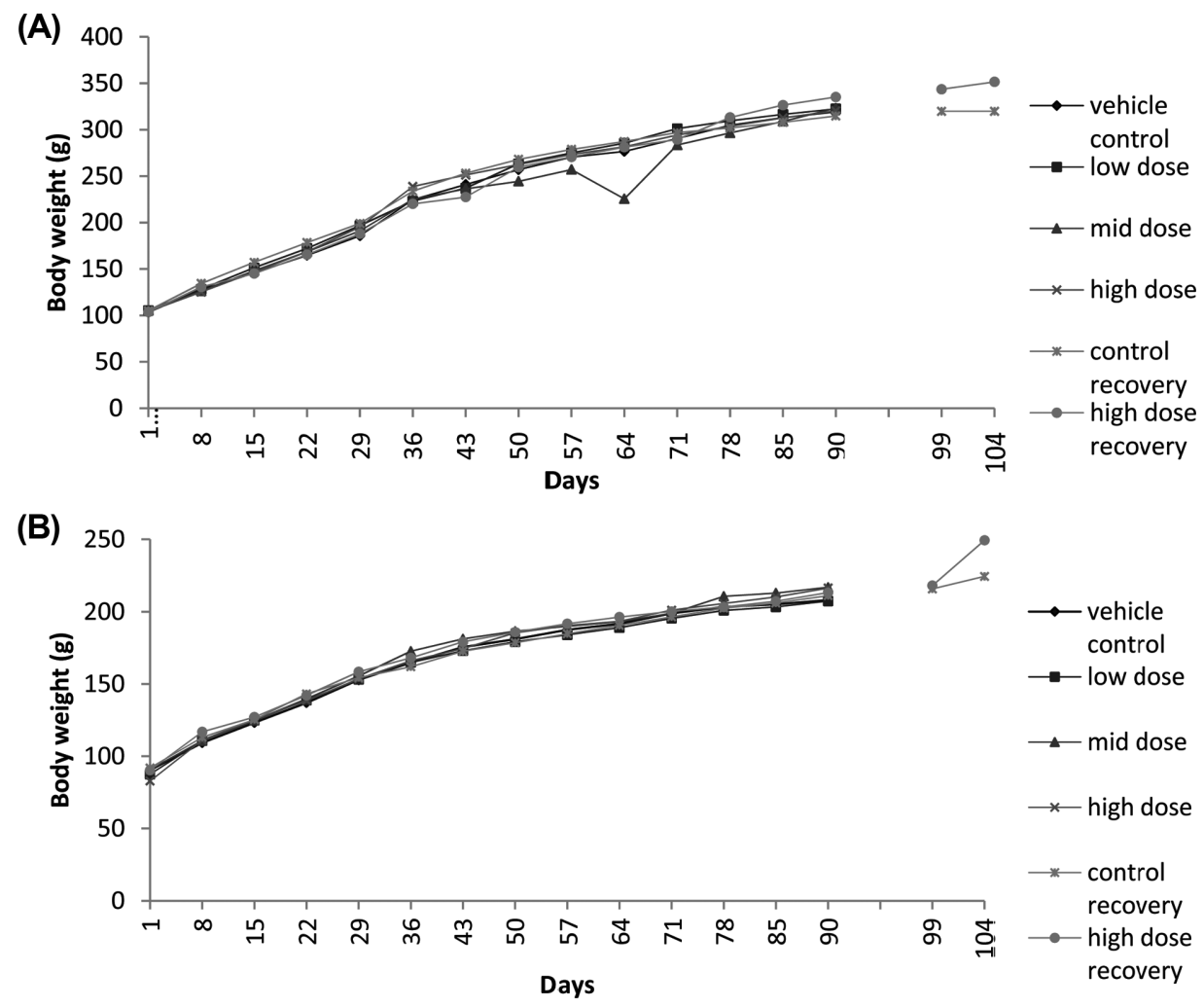

Fig. 2. Effect of graded doses of Tetrahydrocurcumin in 90 days sub-chronic toxicity study. (A) Absolute body weights of male rats in the 90-day subchronic toxicity study. (B) Absolute body weight of female rats in the 90-day subchronic toxicity study. 
ery groups on day 90 and 104 (14 days after the termination of treatments), respectively. Blood samples were collected early in the day to reduce biological variations caused by circadian rhythm. Immediately before sacrifice, the rats were anesthetized with isoflurane/oxygen and blood samples were drawn in a humane manner via the retro-orbital plexus using a heparinized glass microhematocrit capillary tube and placed into two separate tubes: a tube with and without anticoagulant $\left(\mathrm{K}_{2}\right.$ EDTA) for hematology and clinical biochemistry tests, respectively. Urine sample was collected early in the morning before blood sampling on the day of sacrifice. An ABC Vet hematology analyzer, COBAS Roche 9180 electrolyte analyzer, COBAS C111 clinical chemistry analyzer, and COBAS 411 urine analyzer were used to analyze hematological, electrolyte, clinical chemistry, and urine parameters.

All rats were subjected to complete internal and external examination and the results were recorded. The organs from all animals sacrificed at the scheduled euthanasia were trimmed off for adherent tissue and were weighed wet at the earliest to avoid drying were preserved in neutral buffered formalin for further histopathological exam- ination. Histopathological examination was conducted on the organs of rats in groups G1 and G4.

Reproductive and developmental toxicity study. After an acclimatization period of one week, the rats were grouped using computer-generated randomization. The selected males and females were assigned to the control (G1), low-dose (G2), mid-dose (G3), and high-dose (G4) groups (THC at $0,100,200$, and $400 \mathrm{mg} / \mathrm{kg}$ once daily, respectively), as shown in Table 2 .

A single female was placed with a single male from the same group in a $1: 1$ ratio. Cohabitation was continued until vaginal plug or sperm in the vaginal smear was observed. Subsequently, pregnant females were housed individually until lactation day 4. The day of confirmed mating was designated as Gestation Day (GD) 0. The precoital time of each female was calculated. The duration of gestation (gestation length) was calculated from GD0 to the day of parturition.

During the study period, all animals were checked twice daily for any abnormality, morbidity, and mortality. Clinical signs were also observed daily throughout the experimental period. Individual body weights were recorded

Table 3. Effect of 90 days of exposure to THC on hematological parameters in Wistar rats

\begin{tabular}{|c|c|c|c|c|c|c|c|}
\hline \multirow{2}{*}{ Parameter } & \multirow{2}{*}{ Sex } & \multirow{2}{*}{ G1 control } & \multicolumn{3}{|c|}{ Treated } & \multirow{2}{*}{$\begin{array}{l}\text { G1R control } \\
\text { recovery }\end{array}$} & \multirow{2}{*}{$\begin{array}{c}\text { G4R high } \\
\text { dose recovery }\end{array}$} \\
\hline & & & G2 low dose & G3 mid dose & G4 high dose & & \\
\hline \multirow{2}{*}{ Haemoglobin (g/dL) } & $\mathrm{M}$ & $15.82 \pm 1.10$ & $15.93 \pm 1.88$ & $15.14 \pm 1.36$ & $15.61 \pm 1.26$ & $15.48 \pm 0.82$ & $16.12 \pm 1.18$ \\
\hline & $\mathrm{F}$ & $15.76 \pm 1.12$ & $15.64 \pm 1.55$ & $15.34 \pm 1.84$ & $15.63 \pm 1.54$ & $14.60 \pm 0.72$ & $15.42 \pm 1.68$ \\
\hline \multirow{2}{*}{ Haematocrit (\%) } & M & $47.7 \pm 2.92$ & $48.37 \pm 5.89$ & $45.81 \pm 5.22$ & $47.90 \pm 3.94$ & $48.74 \pm 3.09$ & $50.54 \pm 3.47$ \\
\hline & $\mathrm{F}$ & $47.97 \pm 3.94$ & $46.72 \pm 4.85$ & $45.8 \pm 3.92$ & $47.08 \pm 5.19$ & $45.12 \pm 2.10$ & $47.32 \pm 5.00$ \\
\hline \multirow{2}{*}{$\mathrm{RBC}\left(10^{6}\right.$ cells $\left./ \mathrm{mm}^{3}\right)$} & M & $8.91 \pm 0.70$ & $8.94 \pm 0.97$ & $8.46 \pm 1.00$ & $9.04 \pm 0.49$ & $9.24 \pm 0.70$ & $9.45 \pm 0.64$ \\
\hline & $\mathrm{F}$ & $8.78 \pm 0.84$ & $8.53 \pm 0.98$ & $8.36 \pm 0.76$ & $8.73 \pm 0.91$ & $8.14 \pm 0.52$ & $8.41 \pm 1.22$ \\
\hline \multirow{2}{*}{ WBC $\left(10^{3}\right.$ cells $\left./ \mu \mathrm{L}\right)$} & M & $7.68 \pm 2.12$ & $8.06 \pm 1.15$ & $7.48 \pm 1.73$ & $7.11 \pm 1.28$ & $7.40 \pm 0.47$ & $8.22 \pm 0.85$ \\
\hline & $\mathrm{F}$ & $7.17 \pm 1.21$ & $6.42 \pm 0.71$ & $7.25 \pm 1.45$ & $6.74 \pm 1.98$ & $6.42 \pm 1.75$ & $6.00 \pm 1.93$ \\
\hline \multirow{2}{*}{ MCV (fL) } & M & $53.70 \pm 3.06$ & $54.10 \pm 2.18$ & $54.40 \pm 4.38$ & $52.90 \pm 2.73$ & $53.00 \pm 0.71$ & $53.40 \pm 1.14$ \\
\hline & $\mathrm{F}$ & $54.70 \pm 1.57$ & $54.80 \pm 1.40$ & $54.60 \pm 2.07$ & $53.90 \pm 1.45$ & $55.40 \pm 1.95$ & $56.60 \pm 2.51$ \\
\hline \multirow{2}{*}{$\mathrm{MCH}(\mathrm{pg})$} & M & $17.82 \pm 1.14$ & $17.85 \pm 0.84$ & $17.99 \pm 1.70$ & $17.31 \pm 0.81$ & $16.74 \pm 0.48$ & $17.04 \pm 0.55$ \\
\hline & $\mathrm{F}$ & $18.00 \pm 0.98$ & $18.39 \pm 0.76$ & $18.33 \pm 0.75$ & $17.95 \pm 0.73$ & $17.92 \pm 0.64$ & $18.46 \pm 0.78$ \\
\hline \multirow{2}{*}{ MCHC (g/dL) } & M & $33.13 \pm 0.58$ & $32.99 \pm 0.55$ & $33.03 \pm 0.91$ & $32.64 \pm 0.50$ & $31.76 \pm 0.59$ & $31.84 \pm 0.40$ \\
\hline & $\mathrm{F}$ & $32.91 \pm 1.07$ & $33.53 \pm 0.93$ & $33.46 \pm 0.86$ & $33.29 \pm 1.01$ & $32.32 \pm 0.51$ & $32.48 \pm 0.63$ \\
\hline \multirow{2}{*}{ Platelet $\left(10^{3}\right.$ cells $\left./ \mu \mathrm{L}\right)$} & $M^{*}$ & $536.70 \pm 97.62$ & $581.80 \pm 99.88$ & $600.00 \pm 117.01$ & $464.40 \pm 104.12$ & $620.80 \pm 93.55$ & $607.2 \pm 163.12$ \\
\hline & $\mathrm{F}$ & $548.60 \pm 147.31$ & $610.70 \pm 132.99$ & $594.20 \pm 74.23$ & $610.30 \pm 116.34$ & $681.60 \pm 137.31$ & $651.80 \pm 150.23$ \\
\hline \multirow{2}{*}{ Clotting time (sec) } & M & $67.50 \pm 29.37$ & $63.00 \pm 24.29$ & $67.50 \pm 23.72$ & $76.50 \pm 29.54$ & $63.00 \pm 32.52 *$ & $69.00 \pm 39.12^{*}$ \\
\hline & $\mathrm{F}$ & $54.00 \pm 10.49$ & $60.00 \pm 12.25$ & $64.50 \pm 28.33$ & $61.50 \pm 35.67$ & $54.00 \pm 22.75$ & $36.00 \pm 8.22$ \\
\hline \multirow{2}{*}{ Lymphocytes (\%) } & M & $41.80 \pm 7.38$ & $46.10 \pm 6.03$ & $43.20 \pm 7.30$ & $44.00 \pm 5.52$ & $40.40 \pm 4.93$ & $43.20 \pm 1.92$ \\
\hline & $\mathrm{F}^{*}$ & $39.80 \pm 5.16$ & $39.40 \pm 5.34$ & $47.30 \pm 8.00$ & $43.50 \pm 3.37$ & $39.40 \pm 7.09$ & $34.20 \pm 5.07$ \\
\hline \multirow{2}{*}{ Neutrophils (\%) } & M & $43.90 \pm 8.03$ & $40.50 \pm 5.85$ & $42.80 \pm 9.13$ & $43.20 \pm 8.19$ & $47.00 \pm 5.43$ & $44.20 \pm 1.64$ \\
\hline & $\mathrm{F}$ & $47.70 \pm 4.32$ & $48.90 \pm 5.93$ & $40.10 \pm 9.10$ & $42.70 \pm 4.35$ & $48.40 \pm 7.37$ & $55.20 \pm 6.38$ \\
\hline \multirow{2}{*}{ Monocytes (\%) } & $M^{*}$ & $11.00 \pm 1.41$ & $10.10 \pm 2.28$ & $9.30 \pm 1.25$ & $8.60 \pm 2.07$ & $9.00 \pm 1.00$ & $9.40 \pm 1.52$ \\
\hline & $\mathrm{F}^{*}$ & $8.80 \pm 1.32$ & $8.10 \pm 2.69$ & $9.00 \pm 1.33$ & $10.10 \pm 1.52$ & $9.00 \pm 1.41$ & $6.60 \pm 1.82$ \\
\hline \multirow{2}{*}{ Eosinophils (\%) } & M & $3.30 \pm 1.06$ & $3.30 \pm 1.77$ & $4.70 \pm 1.77$ & $4.20 \pm 2.30$ & $3.60 \pm 2.30$ & $3.20 \pm 1.92$ \\
\hline & $\mathrm{F}$ & $3.70 \pm 1.49$ & $3.60 \pm 1.71$ & $3.60 \pm 1.51$ & $3.70 \pm 1.57$ & $3.20 \pm 1.48$ & $4.00 \pm 1.22$ \\
\hline \multirow{2}{*}{ Basophils (\%) } & M & $0.00 \pm 0.00$ & $0.00 \pm 0.00$ & $0.00 \pm 0.00$ & $0.00 \pm 0.00$ & $0.00 \pm 0.00$ & $0.00 \pm 0.00$ \\
\hline & $\mathrm{F}$ & $0.00 \pm 0.00$ & $0.00 \pm 0.00$ & $0.00 \pm 0.00$ & $0.00 \pm 0.00$ & $0.00 \pm 0.00$ & $0.00 \pm 0.00$ \\
\hline
\end{tabular}

$\mathrm{n}=10$, values are in mean \pm SD. *Significant when $p \leq 0.005$. 
during acclimatization, at Day 1 of treatment, at least once weekly during the remainder of the treatment period, and at termination. All dams were weighed on GD 0, 7, 14, and 20 , as well as on lactation days 0 and 4 . Feed consumption per cage was calculated once weekly by the weight of feed left and feed fed.

Complete internal and external necropsy were conducted in all groups and the results were recorded. The organs from animals sacrificed at the scheduled euthanasia were preserved in neutral buffered formalin for histopathological examination as described earlier.

Statistical analysis. Data were subjected to statistical analysis using the Stat Plus program. The data of each group were analyzed by analysis of variance (ANOVA) and expressed as mean \pm standard deviation (SD). $T$-test was used to compare the difference between the treated and control groups. The statistical significance of the differences between groups were determined by one-way ANOVA. All analyses and comparisons were evaluated at a probability level of $5 \%(p \leq 0.05)$.

\section{RESULTS}

In the 90-day subchronic toxicity study, mortality and abnormal clinical signs were not observed in male and female rats in the groups treated with up to $400 \mathrm{mg} / \mathrm{kg} /$ day THC. There was no change in body weight, body weight gain percentage (Fig. 2A, 2B), and food consumption between the THC-treated and control groups. There was no significant change in neurological and hematological parameters (Table 3), clinical chemistry (Table 4), and

Table 4. Effect of 90 days of exposure to THC on clinical chemistry parameters in Wistar rats

\begin{tabular}{|c|c|c|c|c|c|c|c|}
\hline \multirow{2}{*}{ Parameter } & \multirow{2}{*}{ Sex } & \multirow{2}{*}{ G1 control } & \multicolumn{3}{|c|}{ Treated } & \multirow{2}{*}{$\begin{array}{l}\text { G1 R control } \\
\text { recovery }\end{array}$} & \multirow{2}{*}{$\begin{array}{c}\text { G4 R high } \\
\text { dose recovery }\end{array}$} \\
\hline & & & G2 low dose & G3 mid dose & G4 high dose & & \\
\hline \multirow{2}{*}{ Total protein $(\mathrm{g} / \mathrm{L})$} & $\mathrm{M}$ & $83.90 \pm 17.72$ & $84.34 \pm$ & $86.26 \pm 19.72$ & $88.32 \pm 14.69$ & $87.04 \pm 1.60$ & $84.48 \pm 5.72$ \\
\hline & $\mathrm{F}$ & $89.27 \pm 19.26$ & $94.36 \pm 18.93$ & $95.50 \pm 17.75$ & $93.89 \pm 20.25$ & $90.62 \pm 4.05$ & $87.76 \pm 5.86$ \\
\hline \multirow{2}{*}{ Albumin $(\mathrm{g} / \mathrm{L})$} & M & $39.77 \pm 3.66$ & $40.01 \pm 1.95$ & $39.56 \pm 2.32$ & $39.93 \pm 4.39$ & $51.96 \pm 3.09$ & $52.75 \pm 2.91$ \\
\hline & $\mathrm{F}$ & $44.05 \pm 3.15$ & $43.43 \pm 3.87$ & $42.67 \pm 4.30$ & $43.08 \pm 5.62$ & $58.94 \pm 4.17$ & $57.40 \pm 4.06$ \\
\hline \multirow{2}{*}{ ALT (U/L) } & M & $46.10 \pm 15.99$ & $51.88 \pm 24.02$ & $38.88 \pm 8.35$ & $59.52 \pm 37.65$ & $49.64 \pm 8.11$ & $43.56 \pm 9.13$ \\
\hline & $\mathrm{F}$ & $38.59 \pm 7.25$ & $33.42 \pm 5.26$ & $36.95 \pm 5.96$ & $34.32 \pm 11.35$ & $40.48 \pm 11.71$ & $43.32 \pm 11.11$ \\
\hline \multirow{2}{*}{ AST (U/L) } & M & $98.49 \pm 24.11$ & $111.66 \pm 35.77$ & $88.49 \pm 15.85$ & $113.18 \pm 34.90$ & $103.48 \pm 11.63$ & $100.46 \pm 16.49$ \\
\hline & $\mathrm{F}$ & $100.83 \pm 14.52$ & $96.74 \pm 10.53$ & $98.15 \pm 13.65$ & $99.07 \pm 26.04$ & $98.48 \pm 10.07$ & $101.78 \pm 19.50$ \\
\hline \multirow{2}{*}{$\operatorname{ALP}(\mathrm{U} / \mathrm{L})$} & M & $115.13 \pm 27.96$ & $108.88 \pm 19.33$ & $142.10 \pm 54.52$ & $109.93 \pm 24.99$ & $98.28 \pm 16.77$ & 99.00 \\
\hline & $\mathrm{F}$ & $93.74 \pm 36.53$ & $102.63 \pm 47.84$ & $89.53 \pm 45.96$ & $80.01 \pm 33.28$ & $68.80 \pm 18.63$ & $56.18 \pm 15.36$ \\
\hline \multirow{2}{*}{ Total bilirubin (mg/dL) } & M & $0.05 \pm 0.04$ & $0.04 \pm 0.04$ & $0.05 \pm 0.04$ & $0.05 \pm 0.05$ & $0.03 \pm 0.04$ & $0.03 \pm 0.05$ \\
\hline & $\mathrm{F}$ & $0.09 \pm 0.08$ & $0.04 \pm$ & $0.10 \pm 0.05$ & $0.05 \pm$ & $0.02 \pm 0.03$ & $0.03 \pm 0.05$ \\
\hline \multirow{2}{*}{ GGT (U/L) } & M & $0.04 \pm 0.13$ & $0.00 \pm 0.00$ & $0.00 \pm 0.00$ & $0.00 \pm 0.00$ & $0.00 \pm 0.00$ & $0.00 \pm 0.00$ \\
\hline & $\mathrm{F}$ & $0.00 \pm 0.00$ & $0.00 \pm 0.00$ & $0.08 \pm 0.25$ & $0.02 \pm 0.06$ & $0.00 \pm 0.00$ & $0.00 \pm 0.00$ \\
\hline \multirow{2}{*}{ Glucose (mg/dL) } & M & $127.16 \pm 22.61$ & 134.7 & $119.88 \pm$ & 132. & 114.6 & 127.0 \\
\hline & $\mathrm{F}$ & $109.45 \pm 19.84$ & $104.84 \pm 10.07$ & $131.14 \pm 18.23$ & $143.25 \pm 124.38$ & $109.92 \pm 17.73$ & $101.31 \pm 14.83$ \\
\hline \multirow{2}{*}{ Triglycerides (mg/dL) } & M & $39.19 \pm 14.43$ & $40.27 \pm 8.99$ & $44.85 \pm 9.99$ & $46.58 \pm 11.34$ & $63.20 \pm 18.78$ & $58.38 \pm 12.36$ \\
\hline & $\mathrm{F}$ & $48.30 \pm 13.31$ & $35.42 \pm 4.64$ & $47.70 \pm 15.32$ & $45.93 \pm 6.53$ & $78.30 \pm 23.50$ & $86.51 \pm 42.90$ \\
\hline \multirow{2}{*}{ Uric acid $(\mathrm{mg} / \mathrm{dL})$} & M & $35.04 \pm 4.46$ & $40.38 \pm$ & $35.37 \pm$ & 35.1 & $40.92 \pm 4.03$ & $41.35 \pm 5.18$ \\
\hline & $\mathrm{F}$ & $38.87 \pm 8.88$ & $34.95 \pm 5.50$ & $40.53 \pm 8.04$ & $36.26 \pm 6.28$ & $38.36 \pm 5.22$ & $34.11 \pm 4.32$ \\
\hline \multirow{2}{*}{ Creatinine (mg/dL) } & M & $0.31 \pm 0.05$ & $0.34 \pm 0.07$ & $0.33 \pm 0.09$ & $0.32 \pm 0.08$ & $0.34 \pm 0.05$ & $0.35 \pm 0.09$ \\
\hline & $\mathrm{F}$ & $0.39 \pm 0.09$ & $0.40 \pm 0.07$ & $0.38 \pm 0.07$ & $0.38 \pm 0.07$ & $0.41 \pm 0.08$ & $0.38 \pm 0.08$ \\
\hline \multirow{2}{*}{ Total cholesterol* (mg/dL) } & M & $52.13 \pm 9.29$ & $54.46 \pm 9.60$ & $58.95 \pm 7.82$ & $67.85 \pm 11.07$ & $60.81 \pm 8.06$ & $54.92 \pm 10.81$ \\
\hline & $\mathrm{F}$ & $62.25 \pm 12.06$ & $60.17 \pm 12.67$ & $61.99 \pm 11.47$ & $70.11 \pm 8.3$ & $62.28 \pm 16.25$ & $55.66 \pm 12.04$ \\
\hline \multirow{2}{*}{ Phosphate (mg/dL) } & M & $3.80 \pm 6.09$ & $3.89 \pm 6.03$ & $3.97 \pm 6.03$ & $4.06 \pm 6.04$ & $2.29 \pm 0.58$ & $2.17 \pm 0.27$ \\
\hline & $\mathrm{F}$ & $3.81 \pm 6.06$ & $3.90 \pm 6.07$ & $1.99 \pm 0.55$ & $1.94 \pm 0.54$ & $1.96 \pm 0.20$ & $1.82 \pm 0.18$ \\
\hline \multirow{2}{*}{ BUN (mg/dL) } & M & $74.98 \pm 9.54$ & $86.42 \pm 12.96$ & $75.29 \pm 16.17$ & $75.29 \pm 16.17$ & $87.58 \pm 8.62$ & $88.49 \pm 11.07$ \\
\hline & $\mathrm{F}$ & $83.19 \pm 19.01$ & $74.80 \pm 11.77$ & $86.74 \pm 17.21$ & $77.60 \pm 13.44$ & $82.09 \pm 11.18$ & $73.00 \pm 9.25$ \\
\hline \multirow{2}{*}{ Sodium $(\mathrm{mmol} / \mathrm{L})$} & M & $138.30 \pm 1.83$ & $137.70 \pm 1.34$ & $138.40 \pm 1.07$ & $138.40 \pm 1.58$ & $139.00 \pm 1.58$ & $139.00 \pm 1.22$ \\
\hline & $\mathrm{F}$ & $137.80 \pm 1.48$ & $138.20 \pm 0.79$ & $137.00 \pm 0.94$ & $138.50 \pm 1.18$ & $139.40 \pm 6.15$ & $136.00 \pm 2.55$ \\
\hline \multirow[b]{2}{*}{ Potassium (mmol/L) } & M & $5.61 \pm 0.80$ & $5.44 \pm 0.48$ & $5.38 \pm 0.33$ & $5.07 \pm 0.63$ & $5.38 \pm 0.28$ & $5.22 \pm 0.43$ \\
\hline & $\mathrm{F}$ & $5.15 \pm 0.47$ & $5.21 \pm 0.43$ & $5.17 \pm 0.42$ & $5.09 \pm 0.36$ & $4.92 \pm 0.55$ & $5.06 \pm 0.53$ \\
\hline \multirow{2}{*}{ Calcium (mmol/L) } & M & $1.29 \pm 0.05$ & $1.30 \pm 0.05$ & $1.32 \pm 0.04$ & $1.31 \pm 0.03$ & $1.28 \pm 0.03$ & $1.28 \pm 0.07$ \\
\hline & $\mathrm{F}$ & $1.30 \pm 0.07$ & $1.32 \pm 0.05$ & $1.31 \pm 0.05$ & $1.31 \pm 0.05$ & $1.31 \pm 0.07$ & $1.30 \pm 0.05$ \\
\hline
\end{tabular}

$\mathrm{n}=10$, values are in mean \pm SD. *Significant when $p \leq 0.005$. 
Table 5. Effect of 90 days of exposure to THC on urine parameters in Wistar rats

\begin{tabular}{cllccccccc}
\hline \hline \multirow{2}{*}{ Groups } & \multicolumn{7}{c}{ Parameters } \\
\cline { 2 - 10 } & Sex & Appearance & Volume & $\begin{array}{c}\text { Specific } \\
\text { gravity }\end{array}$ & pH & Leukocytes & Protein & Glucose & Erythrocyte \\
\hline \multirow{2}{*}{ G1 vehicle } & $\mathrm{M}$ & Clear & $7.01 \pm 0.53$ & $1.02 \pm 0.01$ & $7.00 \pm 0.58$ & $132.50 \pm 131.26$ & $45.00 \pm 25.82$ & $0.00 \pm 0.00$ & $89.50 \pm 90.20$ \\
& $\mathrm{~F}$ & Clear & $7.05 \pm 0.60$ & $1.02 \pm 0.00$ & $7.00 \pm 0.75$ & $55.00 \pm 38.73$ & $40.00 \pm 24.15$ & $0.00 \pm 0.00$ & $44.00 \pm 58.73$ \\
G2 low dose & $\mathrm{M}$ & Clear to turbid & $7.39 \pm 0.55$ & $1.02 \pm 0.00$ & $7.05 \pm 1.04$ & $220.00 \pm 193.22$ & $72.50 \pm 34.25$ & $0.00 \pm 0.00$ & $52.00 \pm 54.68$ \\
& $\mathrm{~F}$ & Clear to cloudy & $6.57 \pm 0.44$ & $1.02 \pm 0.00$ & $7.25 \pm 0.86$ & $95.00 \pm 146.63$ & $20.00 \pm 22.97$ & $0.00 \pm 0.00$ & $34.50 \pm 42.72$ \\
G3 mid dose & $\mathrm{M}$ & Clear & $7.37 \pm 0.37$ & $1.01 \pm 0.01$ & $7.25 \pm 0.72$ & $77.50 \pm 36.23$ & $30.00 \pm 15.81$ & $0.00 \pm 0.00$ & $21.50 \pm 17.17$ \\
& $\mathrm{~F}$ & Clear to cloudy & $6.84 \pm 0.48$ & $1.02 \pm 0.00$ & $7.20 \pm 0.86$ & $95.00 \pm 146.63$ & $70.00 \pm 152.66$ & $0.00 \pm 0.00$ & $39.70 \pm 59.08$ \\
G4 high dose & $\mathrm{M}$ & Clear to cloudy & $7.22 \pm 0.94$ & $1.02 \pm 0.00$ & $6.95 \pm 0.86$ & $172.50 \pm 174.18$ & $27.50 \pm 18.45$ & $0.00 \pm 0.00$ & $27.50 \pm 43.86$ \\
& $\mathrm{~F}$ & Clear & $6.55 \pm 1.08$ & $1.02 \pm 0.01$ & $6.95 \pm 0.80$ & $55.00 \pm 38.73$ & $30.00 \pm 25.73$ & $0.00 \pm 0.00$ & $12.50 \pm 9.50$ \\
\hline
\end{tabular}

M-male, F-female.

Table 6. Effect of 90 days of exposure to THC on absolute organ weight in Wistar rats

\begin{tabular}{lllccccc}
\hline \hline \multirow{2}{*}{ Organs } & Sex & G1 control & \multicolumn{3}{c}{ Treated } & $\begin{array}{c}\text { G1R control } \\
\text { recovery }\end{array}$ & $\begin{array}{c}\text { G4R high } \\
\text { dose recovery }\end{array}$ \\
\cline { 5 - 6 } Liver & M & $8.60 \pm 1.69$ & $8.81 \pm 1.51$ & $8.47 \pm 1.87$ & $8.74 \pm 1.51$ & $8.53 \pm 0.40$ & $9.69 \pm 1.92$ \\
& F & $5.56 \pm 0.60$ & $5.34 \pm 0.57$ & $5.68 \pm 1.22$ & $6.03 \pm 0.88$ & $6.92 \pm 1.80$ & $7.85 \pm 2.42$ \\
Kidney & $\mathrm{M}$ & $2.05 \pm 0.26$ & $2.08 \pm 0.29$ & $1.98 \pm 0.36$ & $2.16 \pm 0.34$ & $1.93 \pm 0.15$ & $2.20 \pm 0.38$ \\
& $\mathrm{~F}$ & $1.25 \pm 0.10$ & $1.24 \pm 0.16$ & $1.28 \pm 0.17$ & $1.35 \pm 0.15$ & $1.54 \pm 0.26$ & $3.08 \pm 3.25$ \\
Heart & $\mathrm{M}$ & $1.06 \pm 0.20$ & $1.79 \pm 2.35$ & $0.97 \pm 0.12$ & $0.99 \pm 0.14$ & $0.99 \pm 0.12$ & $1.06 \pm 0.11$ \\
& $\mathrm{~F}$ & $0.72 \pm 0.06$ & $0.70 \pm 0.08$ & $0.73 \pm 0.10$ & $0.70 \pm 0.06$ & $0.83 \pm 0.16$ & $0.99 \pm 0.24$ \\
Spleen & $\mathrm{M}$ & $1.09 \pm 0.25$ & $1.14 \pm 0.25$ & $1.02 \pm 0.27$ & $1.12 \pm 0.28$ & $0.86 \pm 0.55$ & $1.03 \pm 0.14$ \\
& $\mathrm{~F}$ & $0.82 \pm 0.24$ & $0.74 \pm 0.10$ & $0.83 \pm 0.16$ & $0.69 \pm 0.10$ & $0.98 \pm 0.32$ & $1.07 \pm 0.34$ \\
Adrenal & $\mathrm{M}$ & $0.04 \pm 0.01$ & $0.05 \pm 0.01$ & $0.04 \pm 0.01$ & $0.05 \pm 0.01$ & $0.05 \pm 0.01$ & $0.11 \pm 0.14$ \\
& $\mathrm{~F}$ & $0.10 \pm 0.14$ & $0.05 \pm 0.01$ & $0.05 \pm 0.01$ & $0.09 \pm 0.12$ & $0.04 \pm 0.01$ & $0.07 \pm 0.02$ \\
Brain & $\mathrm{M}$ & $1.82 \pm 0.13$ & $1.85 \pm 0.12$ & $1.85 \pm 0.15$ & $1.91 \pm 0.09$ & $1.69 \pm 0.20$ & $1.95 \pm 0.18$ \\
& $\mathrm{~F}$ & $1.68 \pm 0.18$ & $1.82 \pm 0.09$ & $1.70 \pm 0.15$ & $1.72 \pm 0.16$ & $1.83 \pm 0.20$ & $1.93 \pm 0.15$ \\
Thymus & $\mathrm{M}$ & $0.38 \pm 0.09$ & $0.31 \pm 0.08$ & $0.34 \pm 0.08$ & $0.42 \pm 0.15$ & $0.30 \pm 0.12$ & $0.34 \pm 0.08$ \\
Testes & $\mathrm{F}$ & $0.34 \pm 0.07$ & $0.31 \pm 0.06$ & $0.26 \pm 0.10$ & $0.28 \pm 0.12$ & $0.36 \pm 0.07$ & $0.72 \pm 0.75$ \\
Ovaries & $\mathrm{M}$ & $3.23 \pm 0.40$ & $3.11 \pm 0.29$ & $2.97 \pm 0.19$ & $3.11 \pm 0.62$ & $3.24 \pm 0.36$ & $3.36 \pm 0.21$ \\
Uterus & $\mathrm{F}$ & $0.33 \pm 0.35$ & $0.19 \pm 0.26$ & $0.10 \pm 0.02$ & $0.10 \pm 0.02$ & $0.12 \pm 0.01$ & $0.33 \pm 0.35$ \\
Epididymus & $\mathrm{F}$ & $0.62 \pm 0.19$ & $0.19 \pm 0.26$ & $0.57 \pm 0.19$ & $0.57 \pm 0.17$ & $0.70 \pm 0.35$ & $0.69 \pm 0.43$ \\
\hline
\end{tabular}

$\mathrm{n}=10$, values are in mean \pm SD. ${ }^{*}$ Significant when $p \leq 0.005$.

urine parameters (Table 5). The absolute and relative organ weights (Table 6) in all treated groups were comparable to those of the control group, and the gross histology of all THC-treated groups also showed no lesion.

In the reproductive and developmental toxicity study, there was no treatment-related abnormal clinical signs and mortality in male and female rats treated with up to 400 $\mathrm{mg} / \mathrm{kg} /$ day THC. Feed consumption, body weight (Fig. $3 \mathrm{~A}, 3 \mathrm{~B})$, and absolute and relative organ weights of male and female rats in all treated groups were comparable to those of the control. There were no treatment-related gross pathological and histopathological lesions (Table 7) in the general organs and tissues as well as the reproductive organs and tissues of male and female animals. Precoital and gestation length was not affected by treatment. Feed consumption, net body weight gain, and changes in body weight of the dams during the gestation period were comparable to those in the lactation period. There was no treatment-related effect on all reproductive parameters and indices (Table 8).

\section{DISCUSSION}

Curcumin is a purified crystal obtained through the solvent extraction of Curcuma longa rhizomes (3). The white THC is a hydrogenated metabolite of curcumin (13). It has antidiabetic, neuroprotective, and anticancer activities along with beneficial effects in fibrotic liver disease $(12,25,26)$. The present study showed the subchronic and reproductive/developmental toxicity profiles of THC in rats.

In the 90-day subchronic toxicity study, THC at doses of up to $400 \mathrm{mg} / \mathrm{kg}$ caused no mortality or adverse effect 

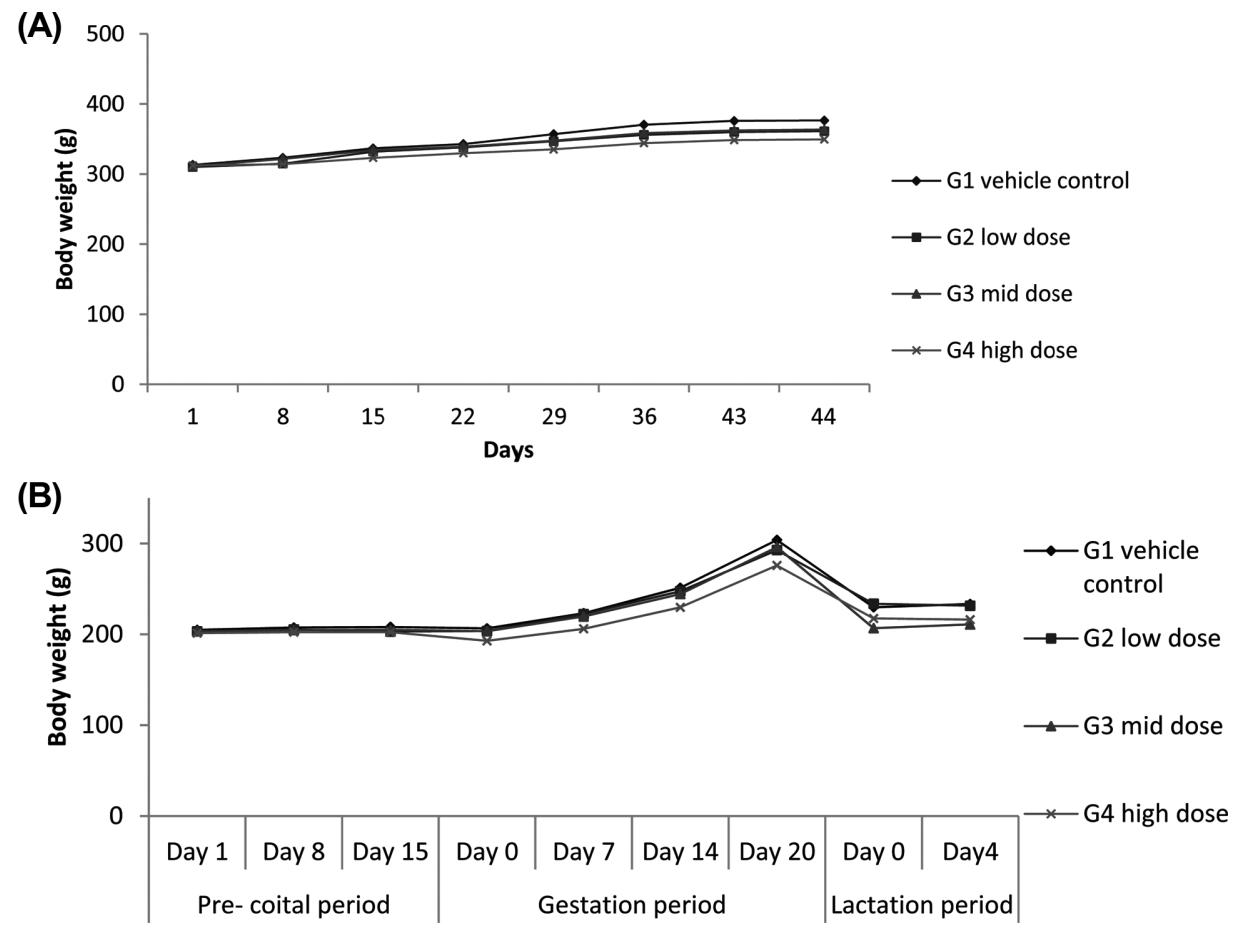

Fig. 3. Effect of graded doses of Tetrahydrocurcumin in reproductive/developmental toxicity study. (A) Average body weights of male rats in the reproductive/developmental toxicity study. (B) Body weight of female rats in the reproductive/developmental toxicity study.

Table 7. Effects of THC on rat reproduction and development

\begin{tabular}{|c|c|c|c|c|c|c|c|c|c|c|}
\hline \multirow{2}{*}{$\begin{array}{c}\text { Group } \\
\text { Organ/lesions }\end{array}$} & \multicolumn{5}{|c|}{ G1 control } & \multicolumn{5}{|c|}{ G4 high dose group } \\
\hline & NAD & Minimal & Mild & Moderate & Severe & NAD & Minimal & Mild & Moderate & Severe \\
\hline \multicolumn{11}{|l|}{ Males } \\
\hline \multicolumn{11}{|l|}{ Testes } \\
\hline Testicular atrophy & $10 / 10$ & - & - & - & - & $9 / 10$ & - & - & - & $01 / 10$ \\
\hline $\begin{array}{l}\text { Sperm development } \\
\text { Epididymides }\end{array}$ & $10 / 10$ & - & - & - & - & $9 / 10$ & - & - & - & $01 / 10$ \\
\hline Sperm stasis & $09 / 10$ & $01 / 10$ & - & - & - & $08 / 10$ & $02 / 10$ & - & - & - \\
\hline $\begin{array}{l}\text { Exfoliated Germ cells } \\
\text { Seminal vesicles }\end{array}$ & $07 / 10$ & $03 / 10$ & - & - & - & $10 / 10$ & - & - & - & - \\
\hline $\begin{array}{l}\text { Atrophy } \\
\text { Prostate glands }\end{array}$ & $10 / 10$ & - & - & - & - & $09 / 10$ & - & - & - & $01 / 10$ \\
\hline $\begin{array}{l}\text { Atrophy } \\
\text { Adrenal glands }\end{array}$ & $10 / 10$ & - & $\because$ & - & - & $09 / 10$ & - & - & - & $01 / 10$ \\
\hline $\begin{array}{l}\text { Angiectasis } \\
\text { Females }\end{array}$ & $08 / 10$ & $02 / 10$ & - & - & - & $08 / 10$ & $01 / 10$ & - & $01 / 10$ & - \\
\hline Ovaries & & & & & & & & & & \\
\hline $\begin{array}{l}\text { Angiectasis } \\
\text { Adrenal glands }\end{array}$ & $10 / 10$ & - & - & - & - & $09 / 10$ & - & - & $01 / 10$ & - \\
\hline Angiectasis & $10 / 10$ & - & - & - & - & $9 / 10$ & - & $01 / 10$ & - & - \\
\hline
\end{tabular}

NAD- no abnormality detected.

during both the treatment and recovery periods. Through ophthalmological examination, we observed that the eyes of the rats were normal during the study period. During the treatment and recovery periods, male and female rats in all treated groups showed no treatment-related differ- ence in mean food consumption, mean body weight, and net body weight gain compared to those in the respective controls. Hematological parameters of male and female rats were normal during the course of the treatment. A marginal decrease in platelet count was observed in male 
Table 8. Summary of histopathological findings in the reproductive/developmental study

\begin{tabular}{|c|c|c|c|c|}
\hline \multirow{2}{*}{$\begin{array}{c}\text { Observation } \\
\text { Dosage (units) }\end{array}$} & \multicolumn{4}{|c|}{ Values } \\
\hline & G1 (control) & G2 (low dose) & G3 (mid dose) & G4 (high dose) \\
\hline Pre-coital interval (days) ${ }^{\#}$ & $4.6 \pm 4.3$ & $5.5 \pm 5.0$ & $5.4 \pm 3.8$ & $5.2 \pm 4.4$ \\
\hline Gestation length (day) $^{\#}$ & $22.6 \pm 0.8$ & $22.3 \pm 0.7$ & $22.6 \pm 0.7$ & $22.3 \pm 0.9$ \\
\hline No. of pair started & 10 & 10 & 10 & 10 \\
\hline No. of pregnant/conceived females (confirmed at necropsy) & 10 & 10 & 10 & 10 \\
\hline No. of pregnancies & 10 & 10 & 10 & 10 \\
\hline No. of dams for corpora lutea and implantation count & 10 & 10 & 10 & 10 \\
\hline No. of corpora lutea/dam (mean) & 11.5 & 11.7 & 11.1 & 11.3 \\
\hline No. of implantation/dam (mean) & 10.8 & 10.9 & 10.5 & 10.4 \\
\hline Male mating index $(\%)$ & 100 & 100 & 100 & 100 \\
\hline Male fertility index (\%) & 100 & 100 & 100 & 100 \\
\hline Female mating index (\%) & 100 & 100 & 100 & 100 \\
\hline Female fecundity index (\%) & 100 & 100 & 100 & 100 \\
\hline Female fertility index (\%) & 100 & 100 & 100 & 100 \\
\hline Mean implantation index (\%) & 95.1 & 92.5 & 94.9 & 92.0 \\
\hline Mean pre-implantation loss (\%) & 4.94 & 7.53 & 5.11 & 8.02 \\
\hline Mean post implantation loss & 15.23 & 12.88 & 16.74 & 29.98 \\
\hline Gestation index $(\%)$ & 100 & 100 & 100 & 100 \\
\hline No. of litters/no. of live litters & $10 / 10$ & $10 / 10$ & $9 / 9$ & $10 / 10$ \\
\hline Total no. of pups born/live pups on day 0 & $96 / 92$ & $96 / 96$ & $91 / 90$ & $83 / 69$ \\
\hline No. of live pups at day 4 & 86 & 91 & 89 & 66 \\
\hline Observation of live/dead pups & $4 / 92$ & $0 / 96^{*}$ & $1 / 90$ & $69 / 14$ \\
\hline Mean viable litter size & 9.6 & 9.6 & 10.1 & 8.3 \\
\hline Mean pups body weight on day $0^{\#}$ & $6.1 \pm 0.79$ & $5.9 \pm 0.75$ & $6.1 \pm 0.45$ & $6.1 \pm 0.57$ \\
\hline Mean body weight on day $4^{\#}$ & $10.4 \pm 1.70$ & $10.1 \pm 2.00$ & $9.2 \pm 0.80$ & $9.9 \pm 0.94$ \\
\hline Sex ratio $(\mathrm{m} / \mathrm{f})($ mean $)$ & 1.25 & 1.27 & 1.17 & 1.06 \\
\hline Live birth index $(\%)$ & 95.8 & 100 & 98.9 & 83.1 \\
\hline Day 4 survival index (\%) & 93.5 & 94.8 & 98.9 & 95.7 \\
\hline
\end{tabular}

*Tail cut off was observed in 9 pups of dam no. 35, "values are in mean \pm standard deviation.

rats of group G4, but the decrease was comparable with that of the control. A marginal decrease in monocyte count was also observed in male rats of groups G3 and G4. The clinical chemistry parameters were normal in almost all rats. There were insignificant increases in total cholesterol and triglycerides in males and females, respectively, of G4 and G4R groups, which were considered to be incidental in nature. Sodium level decreased in female rats of G3, but the decrease was comparable to that of the control.

Urine specific gravity, $\mathrm{pH}$, protein, and leucocyte level were in the normal range in both the main and recovery groups. No treatment-induced gross pathological alterations were noted in any of the groups. The absolute and relative weights of the liver, kidneys, adrenals, spleen, heart, thymus, brain, testes, and epididymides of rats in the treated and recovery groups on day 91 and 105, respectively, were comparable to those of the respective controls. There was no major microscopic finding observed, and observed findings were incidental in nature and not related to the test substance. Taken together, the no-observedadverse-effect-level (NOAEL) of THC was $400 \mathrm{mg} / \mathrm{kg} /$ day.

In the reproductive/developmental toxicity study, there was no mortality except for the incidental death of dam
\#55 due to dystocia on GD23. The mean body weight and net body weight gain of male and female rats in the THCtreated group were comparable to those in the control group during the precoital, gestation, and lactation periods. A marginal decrease in the body weight of dams was observed on GD 0,7, and 14. Total number of pups and body weight of pups were not affected by THC.

There was no treatment-related effect on mean litter size, mean viable litter size, live birth index, and day 4 survival index of the pups. A tail deformity was observed in the pups of dam \#35, which was incidental and not dose-related. The precoital interval, gestation length, male fertility, and mating and fecundity index in all treated groups were comparable to those of the control group. There was no treatment-related effect on implantation index and percentage of pre- and post-implantation loss in all treated groups. Microscopic and macroscopic findings of rat organs did not show any treatment-related effect. The microscopic findings observed were incidental, commonly observed in Wistar rats at that age (29), and were of similar severity in all groups. Thus, our results indicated that THC at oral doses of 100,200 , and $400 \mathrm{mg} / \mathrm{kg} / \mathrm{day}$ was safe with respect to the reproduction and development of 
Wistar rats.

We showed, through the 90-day subchronic and reproductive/developmental toxicity studies, that THC at doses of up to $400 \mathrm{mg} / \mathrm{kg}$ did not cause any adverse effect in rats; thus, we concluded that a repeated dose of $400 \mathrm{mg} / \mathrm{kg}$ was safe to rats and could be considered as the NOAEL of THC under our experimental conditions. Therefore, THC remains unclassified under the hazard category of the Globally Harmonized Classification System.

\section{CONFLICT OF INTEREST}

The authors have no conflict of interest to disclose.

Received June 22, 2018; Revised August 14, 2018; Accepted September 7, 2018

\section{REFERENCES}

1. Pandey, A., Gupta, R.K. and Srivasta, R. (2011) Curcumin the yellow magic. Asian J. Appl. Sci., 4, 343-354.

2. Stankovic, I. (2004) Curcumin, Chemical and Technical Assessment (CTA). 61st JECFA, FAO.

3. Prasad, S. and Aggarwal, B.B. (2011) Chapter 13. Turmeric, the golden spice: from traditional medicine to modern medicine in Herbal Medicine: Biomolecular and Clinical Aspects (2nd edition) (Benzie, I.F.F. and Wachtel-Galor, S., Eds.). CRC Press/Taylor \& Francis, Boca Raton. Available from: https://www.ncbi.nlm.nih.gov/books/NBK92752/.

4. Pawar, H., Karde, M., Mundle, N., Jadhav, P. and Mehra, K. (2014) Phytochemical evaluation and curcumin content determination of turmeric rhizomes collected from Bhandara District of Maharashtra (India). Med. Chem., 4, 588591.

5. Chattopadhyay, I., Biswas, K., Bandyopadhyay, U. and Banerjee, R. (2004) Turmeric and curcumin: biological actions and medicinal applications. Current Sci., 87, 44-53.

6. National Center for Biotechnology Information (2005) Tetrahydrodiferuloylmethane. PubChem Compound Database, CID: 124072 [accessed 2018 Jan 5]. Available from: https:// pubchem.ncbi.nlm.nih.gov/compound/.

7. Manjunatha, J.R. and Srinivas, P. (2015) Synthesis of Water Soluble Curcumin Derivatives and Evaluation of Their Bioactive and Anti Amyloid Aggregating Properties. University of Mysore, Sodhaganga. Available from: http:/hdl.handle.net/ 10603/102621/

8. Majeed, M., Pineda, R., Chan, G., Gabriel, T., Dayrit, J., Pelayo, C.A. and Prakash, L. (2010) A randomized, doubleblind, placebo-controlled, comparative study The safety and efficacy of $0.25 \%$ tetrahydrocurcumin (turmeric) cream as depigment agent against $4 \%$ hydroquinone cream. $H \& P C$ Today, 3, 44-46.

9. Randino, R., Grimaldi, M., Persico, M., Santis, A.D., Cini, E., Cabri, W., Riva, A., D’Errico, G., Fattorusso, C., D’Ursi, A.M. and Rodriquez, M. (2016) Investigating the neuroprotective effects of turmeric extract: structural interactions of $\beta$-amyloid peptide with single curcuminoids. Sci. Rep., 6,
38846.

10. Hassaninasab, A., Hashimoto, Y., Tomita-Yokotani, K. and Kobayashi, M. (2011) Discovery of the curcumin metabolic pathway involving a unique enzyme in an intestinal microorganism. PNAS, 108, 6615-6620.

11. Vijaya Saradhi, U.V., Ling, Y., Wang, J., Chiu, M., Schwartz, E.B., Fuchs, J. R., Chan, K.K. and Liu, Z. (2010) A liquid chromatography-tandem mass spectrometric method for quantification of Curcuminoids in cell medium and mouse plasma. J. Chromatogr. B, 878, 3045-3051.

12. Aggarwal, B.B., Deb, L. and Prasad, S. (2015) Curcumin differs from tetrahydrocurcumin for molecular targets, signaling pathways and cellular responses. Molecules, 20, 185205.

13. Pan, M.S., Huang, T.M. and Lin, J.K. (1999) Biotransformation of curcumin through reduction and glucuronidation in mice. Drug Metab. Dispos., 27, 486-494.

14. Vijayan, D., Tintu, I. and Sadasivan, C. (2011) Molecular docking studies of curcumin analogs with phospholipase A2. Interdiscip. Sci. Comput. Life Sci., 3, 189-197.

15. Somparn, P., Phisalaphong, C., Nakornchai, S., Unchern, S. and Morales, N.P. (2007) Comparative antioxidant activities of curcumin and its demethoxy and hydrogenated derivatives. Biol. Pharm. Bull., 30, 74-78.

16. Mukhopadhyay, A., Basu, N., Ghatak, N. and Gujral, P.K. (1982) Anti-inflammatory and irritant activities of curcumin analogs in rats. Agents Actions, 12, 508-515.

17. Murugan, P. and Pari, L. (2007) Influence of tetrahydrocurcumin on erythrocyte membrane bound enzymes and antioxidant status in experimental type 2 diabetic rats. $J$. Ethnopharmacol., 113, 479-486.

18. Pari, L. and Murugan, P. (2007) Antihyperlipidemic effect of curcumin and tetrahydrocurcumin in experimental type 2 diabetic rats. Renal Fail., 29, 881-889.

19. Nakmareong, S., Kukongviriyapan, U., Pakdeechote, P., Donpunha, W., Kukongviriyapan, V., Kongyingyoes, B., Sompamit, K. and Phisalaphong, C. (2011) Antioxidant and vascular protective effects of curcumin and tetrahydrocurcumin in rats with L-NAME-induced hypertension. Naunyn Schmiedebergs Arch. Pharmacol., 383, 519-529.

20. Pari, L. and Murugan, P. (2007) Influence of tetrahydrocurcumin on tail tendon collagen contents and its properties in rats with streptozotocin-nicotinamide-induced type 2 diabetes. Fund. Clin. Pharmacol., 21, 665-671.

21. Sugiyama, Y., Kawakishi, S. and Osawa, T. (1996) Involvement of the beta-diketone moiety in the antioxidative mechanism of tetrahydrocurcumin. Biochem. Pharmacol., 52, 519525.

22. Khopde, S.M., Priyadarsini, K.I., Guha, S.N., Satav, J.G., Venkatesan, P. and Rao, M.N. (2000) Inhibition of radiationinduced lipid peroxidation by tetrahydrocurcumin: possible mechanisms by pulse radiolysis. Biosci. Biotechnol. Biochem., 64, 503-509.

23. Okada, K., Wangpoengtrakul, C., Tanaka, T., Toyokuni, S., Uchida, K. and Osawa, T. (2001) Curcumin and especially tetrahydrocurcumin ameliorate oxidative stress-induced renal injury in mice. J. Nutr., 131, 2090-2095.

24. Naito, M., Wu, X., Nomura, H., Kodama, M., Kato, Y., Kato, Y. and Osawa, T. (2002). The protective effects of tet- 
rahydrocurcumin on oxidative stress in cholesterol-fed rabbits. J. Atheroscler. Thromb., 9, 243-250.

25. Lai, C.S., Wu, J.C., Yu, S.F., Badmaev, V., Nagabhushanam, K., Ho, C.T. and Pan M.H. (2011) Tetrahydrocurcumin is more effective than curcumin in preventing azoxymethaneinduced colon carcinogenesis. Mol. Nutr. Food Res., 55, 1819-1828.

26. Tsai, M.-L., Tsai, S.-P. and Ho, C.-T. (2017) Tetrahydrocurcumin attenuates carbon tetrachloride-induced hepatic fibrogenesis by inhibiting the activation and autophagy of hepatic stellate cells. J. Funct. Foods, 36, 418-428.

27. Wu, J.-C., Lai, C.-S., Badmaev, V., Nagabhushanam, K., Ho,
C.T. and Pan, M.H. (2011) Tetrahydrocurcumin, a major metabolite of curcumin, induced autophagic cell death through coordinative modulation of PI3K/Akt-mTOR and MAPK signaling pathways in human leukemia HL-60 cells. Mol. Nutr. Food Res., 55, 1646-1654.

28. Majeed, M., Pande, A., Paliwal, P. and Nagabhushanam, K. (2012) Tetrahydrocurcuminoids. NutriScience Publishers.

29. Kroes, R., Garbis-Berkvens, J.M., de Vries, T. and van Nesselrooy, J.H.J. (1981) Histopathological profile of a Wistar rat stock including a survey of the literature. J. Gerontol., 36, 259-279. 